\title{
The role of dairy products in sustainable diets: modelling nutritional adequacy, financial and environmental impacts
}

\author{
D.A Hobbs ${ }^{1}$, J.A Lovegrove ${ }^{1}$ and D.I Givens ${ }^{2}$ \\ ${ }^{1}$ Hugh Sinclair Unit of Human Nutrition and Institute for Cardiovascular and Metabolic Research and ${ }^{2}$ School of \\ Agriculture, Policy and Development, Department of Food Production and Quality, University of Reading, Reading, \\ United Kingdom
}

Milk and dairy products are important nutritional components of the British diet, providing essential nutrients such as protein, calcium, phosphorus, iodine and vitamin $\mathrm{B}_{12}{ }^{(1)}$. The food system is responsible for around $20 \%$ of all green-house gas emissions (GHGEs), in the UK, with meat and dairy products being the largest individual contributors ${ }^{(2)}$. The aim of this study was to determine the role of dairy products in sustainable diets by modelling nutritional adequacy, financial cost and GHGEs of the UK diets containing high, low and average amounts of dairy products (milk, cheese, yogurt and ice cream).

A predictive response model was created using data from years 1-4 (2008-09 to 2011-12) of the National Diet and Nutrition Survey (NDNS) for men $(n=710)$ aged 19-64 years old ${ }^{(3)}$. Nutrient composition, financial costs and GHGE data for 63 food groups were used to estimate nutritional adequacy, financial costs and GHGEs for the average UK diet, high dairy and low dairy diets. High and low dairy diets were obtained by organising dietary data for quartile of dairy intake, with the highest/lowest quartile representing the high/low dairy diet. GHGEs were calculated using Life Cycle Analysis (LCA) from primary production to retail. The financial cost of foods $(n=3423)$ were obtained from two UK supermarkets (Asda and Waitrose) from June to September 2014. Differences in nutritional contribution between diets were determined using one-way ANOVA with Bonferroni multiple comparisons test.

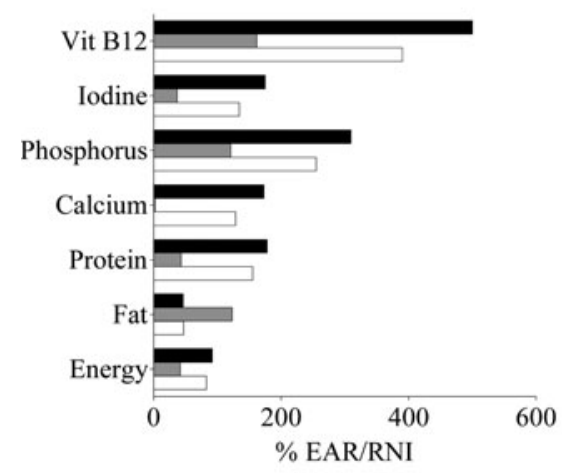

Average UK diet

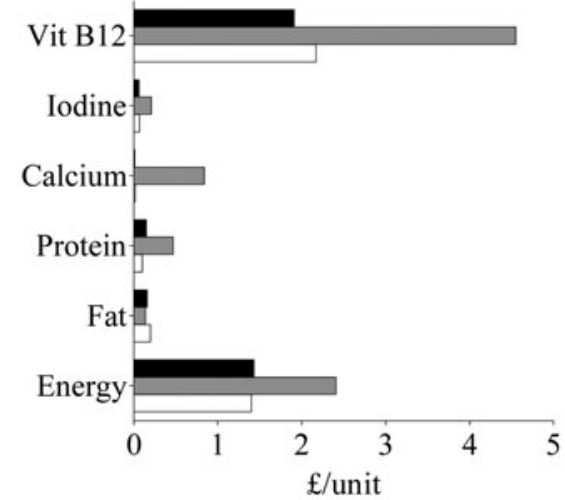

£/unit

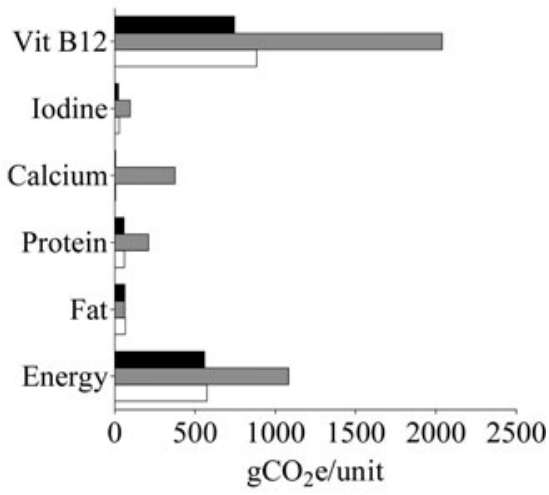

Fig. 1. Nutritional contribution

Fig. 2. Financial cost

Fig. 3. Greenhouse gas emissions

The average, high and low UK dairy diets contained 207, 420 and $54 \mathrm{~g}$ of dairy per day, respectively. The high dairy diet provided significantly more vitamin $\mathrm{B}_{12}(P<0.05)$, iodine $(P<0.05)$, phosphorous $(P<0.01)$, calcium $(P<0.01)$, protein $(P<0.01)$ and energy $(P<0.0001)$ compared with the low dairy diet (Fig 1). The financial cost of the average UK diet, the high dairy and low dairy diets were $£ 12.7 /$ day, $£ 14.4 /$ day and $£ 11.0 /$ day, respectively. The low dairy diet was $69,213,8300,250$ and $138 \%$ more expensive for energy, protein, calcium, iodine and vitamin $\mathrm{B}_{12}$, respectively compared with the high dairy diet (Fig 2). The GHGEs for the average UK diet was 5181, 5601 and $4955 \mathrm{gCO}_{2} \mathrm{e} /$ day, respectively. The GHGEs from the low dairy diet were 94, 266, 8036, 320 and $174 \%$ higher for energy, protein, calcium, iodine and vitamin $\mathrm{B}_{12}$, respectively compared with the high dairy diet (Fig 3 ).

These findings highlight that low dairy consumption $(<90 \mathrm{~g} /$ day) may have nutritional implications and does not reduce GHGEs or lower financial cost per nutrients.

This work was supported by The Dairy Council and DairyCo.

1. Givens DI \& Kliem K (2011) Annu Rev Food Sci Technol. 2, 21-36.

2. Audsley E, Brander M, Chatterton J et al. (2009) WWF-UK

3. Bates B, Lennox A, Prentice A et al. (2014) NDNS (2008/09-2011/12). 